\title{
Lessons Learnt from Cerebrospinal Meningitis Outbreak Surveillance Data-A Case for Public Health Action
}

\author{
Semeeh Akinwale Omoleke ${ }^{1}$, Olatunji Alabi ${ }^{2}$, Yakubu Bashar Usman ${ }^{3}$, \\ Kunle Ijaya ${ }^{4} \&$ Abubakar Abubakar Koko ${ }^{5}$ \\ ${ }^{1}$ Immunisation, Vaccines and Emergencies Unit, World Health Organisation, Nigeria \\ ${ }^{2}$ Demography and Social Statistics, Federal University, Birnin Kebbi, Nigeria \\ ${ }^{3}$ State Public Health Laboratory, Ministry of Health, Kebbi State, Nigeria \\ ${ }^{4}$ World Health Organisation, Kebbi State Office, Nigeria \\ ${ }^{5}$ Department of Medical Services, Ministry of Health, Kebbi State, Nigeria \\ Correspondence: Semeeh Akinwale Omoleke, Immunisation, Vaccines and Emergencies Unit, World Health \\ Organisation, Nigeria. Tel: 234-816-759-7029. E-mail: talk2semeeh@yahoo.co.uk; omolekes@who.int
}

Received: March 9, 2016 Accepted: June 3, 2016 Online Published: June 30, 2016

doi:10.5539/gjhs.v9n2p76 URL: http://dx.doi.org/10.5539/gjhs.v9n2p76

\begin{abstract}
Background: Outbreak of cerebrospinal meningitis (CSM) remains a major public health concern in Nigeria, particularly in northern Nigeria. The paper evaluates the effect of mass vaccination against cerebrospinal meningitis outbreak in 2013 on the incidence rate in 2014 and 2015, and to document lessons learnt from field experiences of the meningitis epidemic surveillance in Kebbi State, North-west, Nigeria.

Methods: The authors analysed cerebrospinal meningitis surveillance data generated from the routine integrated disease surveillance and response (IDSR) programme executed by the Ministry of Health with support from the World Health Organisation (WHO). Cerebrospinal fluid (CSF) samples via lumbar puncture procedures from a small proportion of all suspected cases that met the standard case definitions were collected, and then tested using the rapid agglutination test kits (Pastorex) at the state public health laboratory. The WHO supported the trained Local Government Areas Disease Surveillance and Notification Officers to ensure data quality.

Results: A total of 544 and 1,992 cases were analysed in 2014 and 2015 respectively. In 2014, 14\% CSF samples were taken and $55.1 \%$ tested positive to Neisseria meningitidis type C. Of all the cases in $2014,14 \%$ were reported dead. Further, in 2015, 4\% CSF samples were tested and $83 \%$ were positive to Neisseria meningitidis type C. Of the total 1,992 cases in 2015, 4\% were reported dead. Gender and CSF sample testing significantly predicts survival in $2014(\mathrm{p}<0.05)$.

Conclusion: Desired political will and comprehensive epidemic prevention and control strategies are needed for effective control of seasonal outbreaks of CSM and other epidemic-prone diseases. Need for infrastructural and capacity development of hospital and state public health laboratories for adequate surveillance, testing of samples collected and effective case management cannot be over-emphasized.
\end{abstract}

Keywords: cerebro-spinal meningitis, surveillance, mass vaccination, laboratory, Nigeria

\section{Introduction}

The seasonal epidemic of meningitis within the 'African meningitis belt' has persisted for at least 60years. The pathogenic cause of this epidemic is primarily Neisseria meningitidis ( $\mathrm{Nm}$ ) with most of the epidemic caused by serotype A (Marc LaForce, Ravenscroft, Djingarey, \& Viviani, 2002;WHO, 2010), though, recently W135 has also emerged as a cause of epidemic in some West African countries such as Burkina Faso (Yves et al., 2006). Other bacterial agents such as Streptococcus pneumoniae and Haemophilus influenzae type b also contribute to a much lesser degree to the burden (WHO, 2010).

The meningitis belt extends from Senegal in West Africa to Ethiopia in Eastern Africa and typically between months of December/January and April/May when the prevailing weather condition could be best described as the dry, hot, dusty and windy (Greenwood, 1999; Molesworth, Cuevas, Connor, Morse, \& Thomson, 2003). The epidemic typically tails off with the onset of rain in the month of June when temperature drops; atmospheric 
humidity is higher and less dusty. However, it should be noted that the impact of climate change seems to have altered the duration of this epidemic and the breadth of the meningitis belt. Despite this understanding from spatial epidemiology, advances in anti-microbial therapy, improved medical diagnostic technology and availability of potent vaccines, the epidemic still constitute public health challenges- morbidity, mortality and socio-economic cost, within the meningitis belt, though at varying degrees across countries.

Nigeria, particularly Northern Nigeria, has continually experienced the seasonal outbreaks of meningitis. Further, challenges impeding effective epidemic prevention and control remain in spite of efforts by government and health development partners. Part of the efforts to prevent and control the seasonal epidemic include mass vaccination targeted at serotype A in 2013 and recent effort being made to improve Cerebrospinal Meningitis (CSM) laboratory surveillance for a more robust characterization of the epidemic. Despite some of these interventions, the epidemic has expanded in Nigeria in the last 3years (2013-2015) from 26 states to 29 states (FMOH/WHO, 2016), hence, the need for a better coordinated response and political will to stem the seasonal epidemic.

In the light of the above, this study sets out to explore the relationships between Cerebrospinal Fluid (CSF) microbial analysis and mass vaccination, and to document lessons learnt and proffer recommendations based on field experiences from the last 2 consecutive years of meningitis epidemic in one of the Northwestern states of Nigeria- Kebbi.

\section{Method}

\subsection{Study Setting}

Kebbi State is one of the Sahel Savannah states of northwestern Nigeria. It has international borders with Niger and Benin Republics. It is bordered by 2 other northwestern states-Zamfara and Sokoto, and a north central state-Niger. The state has 21 Local Government Areas (LGAs), 225 political wards and 4 traditional emirates. It has a projected population of 4,394,887 for the year 2016 based on 2006 census population. It is essentially a rural state with majority of the population engaged in agriculture and agro-allied activities.

It has over the years been experiencing meningitis outbreak and the epidemiology often mimic those reported in Niger Republic. It could be best described as the epi-centre of meningitis outbreak in Northwestern Nigeria. Northwestern Nigeria accounted for $80 \%$ of cases reported and $70 \%$ of death (FMOH/WHO, 2016). The Northwest constitutes $25 \%$ of total population of Nigeria, underscoring the public health significance of an epidemic in this part of Nigeria.

\subsection{Data Collection}

Data from this study was generated from the routine integrated disease surveillance and response being done in Nigeria through the Ministry of Health and supported by the World Health Organisation (WHO). Standard electronic template as well as hard copies of line list were generated at the health facility level or designated treatment camps in an epidemic setting from various LGAs. The line list typically contains information about the suspected or confirmed cases of meningitis and these details include name, sex, age address, number of immunization doses, (CSF) sample taken, epidemic week of onset, result/finding (positive or negative test) and outcome (dead or alive).

At the health facility level or treatment camps, there are standard case definitions being used to identify and report the disease. In other words, Cerebrospinal Meningitis is said to be suspected if a patient who is above 30 months presents with acute onset of fever (usually $\geq 38.5^{\circ} \mathrm{C}$ rectal or $38^{\circ} \mathrm{C}$ axillary), headache and any of the following-neck stiffness, loss of consciousness and other meningeal signs or any infant with sudden onset of fever $\geq 38.5^{\circ} \mathrm{C}$ with one of the following signs: neck stiffness or soft neck, bulging fontanelle, convulsion or other meningeal signs. A confirmed case is any case for which the CSF analysis in the laboratory is positive either through rapid tests, by culture or PCR positive while a compatible case is one in which a lumbar puncture produces CSF that is cloudy or purulent for which the laboratory testing is not carried out or carried out and the result is negative. Or any cases with a positive Gram test, whose white blood cell count $>10 / \mathrm{mm}^{3}$ and for which the rapid tests, culture and PCR are negative or not done or any case having evident clinical signs in the context of a meningitis outbreak (WHO, 2016).

Data generated at the Local Government Area (LGA) level is transmitted to the state by a technical officerDisease Surveillance and Notification Officer (LGA DSNO). At this level, WHO officers supporting the LGA also provide technical support and logistics to enhance data quality while the State Epidemiologist supported by the State DSNO collates the data at the state level for onwards transmission to national level, i.e., Federal Ministry of Health (FMOH). Technical support is also provided at this level WHO by Surveillance Officer. 
In practice, especially in Northern Nigeria, doctors who are often supported by Community Health Worker and/or Public Health Nurses receive the patients and manage the cases as per WHO recommended treatment guideline in outbreak setting. In view of the fact that case-based surveillance is being advocated, doctors were and are being encouraged to investigate by taking CSF samples via lumbar puncture procedure from all suspected cases that meet the standard case definitions.

CSF samples were collected from some suspected cases during the outbreaks and were tested using the rapid agglutination test kit (Pastorex) at the state public health laboratory. The CSF samples were maintained in cold temperature from the treatment centres or health facilities to the laboratory using vaccine carriers/giostyle. The State Laboratory Scientist received the samples and conducted tests. Note that in 2014, further test- culture study was done but this was not possible in 2015. The challenges associated with laboratory confirmation and related issues will be elaborated in the discussion section.

Data for year 2014 and 2015 were exported to STATA 12 for the analysis. Data cleaning was done using STATA 12. The data were analysed at univariate and bivariate level of analysis using the appropriate inferential and descriptive statistics. At the univariate level, simple frequency table was used to describe the data for the two years.

\section{Result}

Table 1 showed that a total of 544 cases were reported and analysed in the year 2014 while 1,992 cases were analysed in year 2015. Further, the table showed that more than half of the respondents for the two years were males. Almost three-quarter (74\%) of the sample in year 2014 were aged 2-14 years while more than two-third $(68 \%)$ of the samples in year 2015 were aged 2-14 years.

Figure 1 showed the distribution of suspected and confirmed cases by week of onset of disease for 2014 and 2015. The graph showed that meningitis outbreak data for 2014 peaked at week 12 and 13 as the week of onset of disease while 2015 data peaked at week 8 and 13 .

From Table 1, out of the total sample (suspected and confirmed cases) for the two years, only 14 percent and 4 percent CSF samples were taken for the year 2014 and 2015 respectively. Of the CSF samples taken for both years, more than half $(55.1 \%)$ were positive to $\mathrm{Nm} \mathrm{C}$ in year 2014 while eighty-three percent $(83 \%)$ of the sample for year 2015 were positive to $\mathrm{Nm} \mathrm{C}$. The survival outcome variable for both year showed that fourteen percent $(14 \%)$ of the 544 cases for the year 2014 were reported dead while four percent $(4 \%)$ were reported dead in the year 2015 out of the 1,992 cases.

Table 2 showed the bivariate relationship between the survival outcome and selected characteristics of the samples for both year 2014 and 2015. The test of relationship between survival and sex showed that sex is significantly associated with survival outcome in the year $2014(\mathrm{P}<0.05)$. Further, table 2 showed that CSF sample taken was also associated with survival in the year $2014(\mathrm{P}<0.05)$. However, the three selected characteristics were not significantly associated with survival in the year 2015.

Figure 2 showed the case fatality rate by Local Government Area (LGA) in the 2 year period under review. Generally, case fatality rates decrease across the LGA between 2014 and 2015. For example, the case fatality rate in Aliero and Birnin-Kebbi LGAs decrease from 36.8\% and 21.1\% respectively in 2014 to $29.3 \%$ and $5.2 \%$ respectively in 2015. However, in Bunza, Fakai and Zuru LGAs, case fatality rate increased from $10.5 \%, 0 \%$ and $0 \%$ respectively in 2014 to $27.6 \%, 1.7 \%$ and $12.1 \%$ respectively in 2015 .

Table 1. Percent distribution of cases by socio-demographic and health characteristics, 2014 and 2015

\begin{tabular}{lllll}
\hline Characteristics & $\mathbf{2 0 1 4}$ & & $\mathbf{2 0 1 5}$ & \\
\cline { 2 - 5 } & Frequency & Percentage & Frequency & Percentage \\
\hline Sex & 308 & 56.62 & 1,127 & 56.58 \\
Male & 236 & 43.38 & 865 & 43.42 \\
Female & & & & \\
Age (years) & 32 & 5.88 & 28 & 1.41 \\
less than 2 & 404 & 74.26 & 1,352 & 67.87 \\
$2-14$ & 99 & 18.20 & 478 & 24.00 \\
$15-29$ & 9 & 1.65 & 134 & 6.73 \\
$30+$ & & & & \\
\hline
\end{tabular}




\begin{tabular}{lllll}
\hline CSF Taken? & 467 & 86.00 & 1,917 & 96.23 \\
No & 76 & 14.00 & 75 & 3.77 \\
Yes & & & & \\
No of valid vaccine doses & 204 & 37.50 & 740 & 37.15 \\
0 & 335 & 61.58 & 1,252 & 62.85 \\
1 & 5 & 0.92 & - & - \\
2 & & & & \\
Result & 27 & 39.13 & 12 & - \\
Negative & 1 & 1.45 & - & - \\
Not Tested & 2 & 2.90 & - & - \\
S.PNEUM & 1 & 1.45 & - & 82.86 \\
Positive to Hi B & 38 & 55.07 & 58 & \\
Positive to Nm c & & & & 4.02 \\
Outcome & 75 & 13.79 & 80 & 95.98 \\
Dead & 469 & 86.21 & 1,912 & \\
Alive & & & & \\
\hline
\end{tabular}

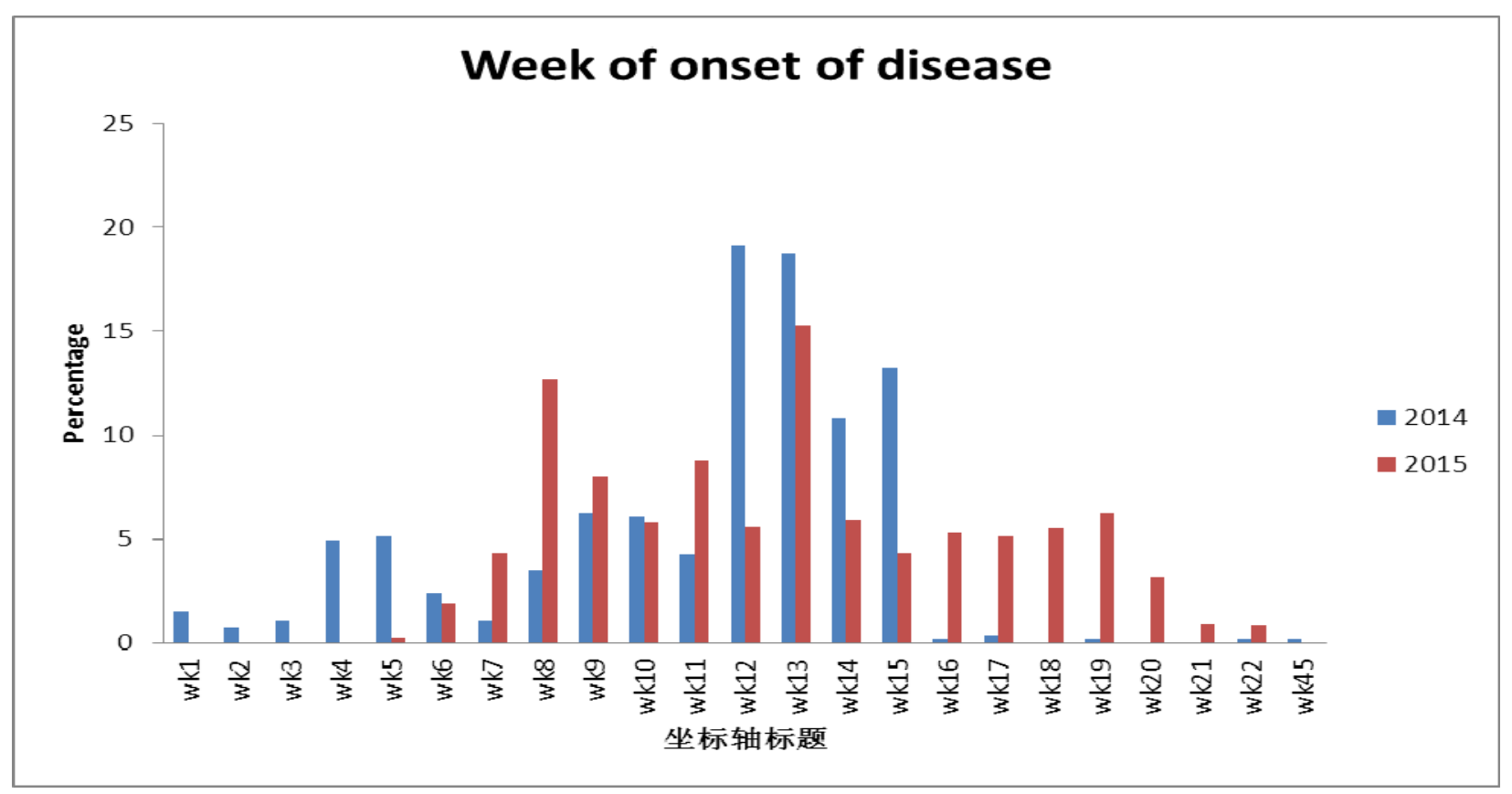

Figure 1. Distribution of respondents by week of onset of disease

Table 2. Bivariate relationship between selected characteristics and survival status

\begin{tabular}{lllllll}
\hline \multirow{2}{*}{ Characteristics } & \multicolumn{5}{l}{$\mathbf{2 0 1 4}(\mathbf{N}=\mathbf{5 4 4})$} & \multicolumn{2}{l}{$\mathbf{2 0 1 5}$} \\
\cline { 2 - 7 } & \multicolumn{2}{l}{ Outcome } & P-value $(\boldsymbol{\chi 2})$ & \multicolumn{2}{l}{ Outcome } & P-value $(\boldsymbol{\chi 2})$ \\
\cline { 2 - 7 } & Alive & Died & & Alive & Died & \\
\hline Sex & 83.44 & 16.56 & & & & $\mathbf{0 . 9 5 2}$ \\
Male & 89.93 & 10.17 & & 96.01 & 3.99 & \\
Female & & & $\mathbf{0 . 8 5 8}$ & 95.95 & 4.05 & \\
Age group & 81.25 & 18.75 & & & & $\mathbf{0 . 0 5 4}$ \\
Less than 2years & 86.39 & 13.61 & & 89.29 & 10.71 & \\
2-14years & & & 96.30 & 3.70 & \\
\hline
\end{tabular}




\begin{tabular}{lcccccc}
\hline 15-29years & 86.87 & 13.13 & & 94.77 & 5.23 & \\
30years and above & 88.89 & 11.11 & & 98.51 & 1.49 & \\
CSF Taken & & & $\mathbf{0 . 0 2 2}$ & & & $\mathbf{0 . 2 2 8}$ \\
No & 85.01 & 14.99 & & 95.88 & 4.12 & \\
Yes & 94.74 & 5.26 & & 98.67 & 1.33 & \\
\hline
\end{tabular}

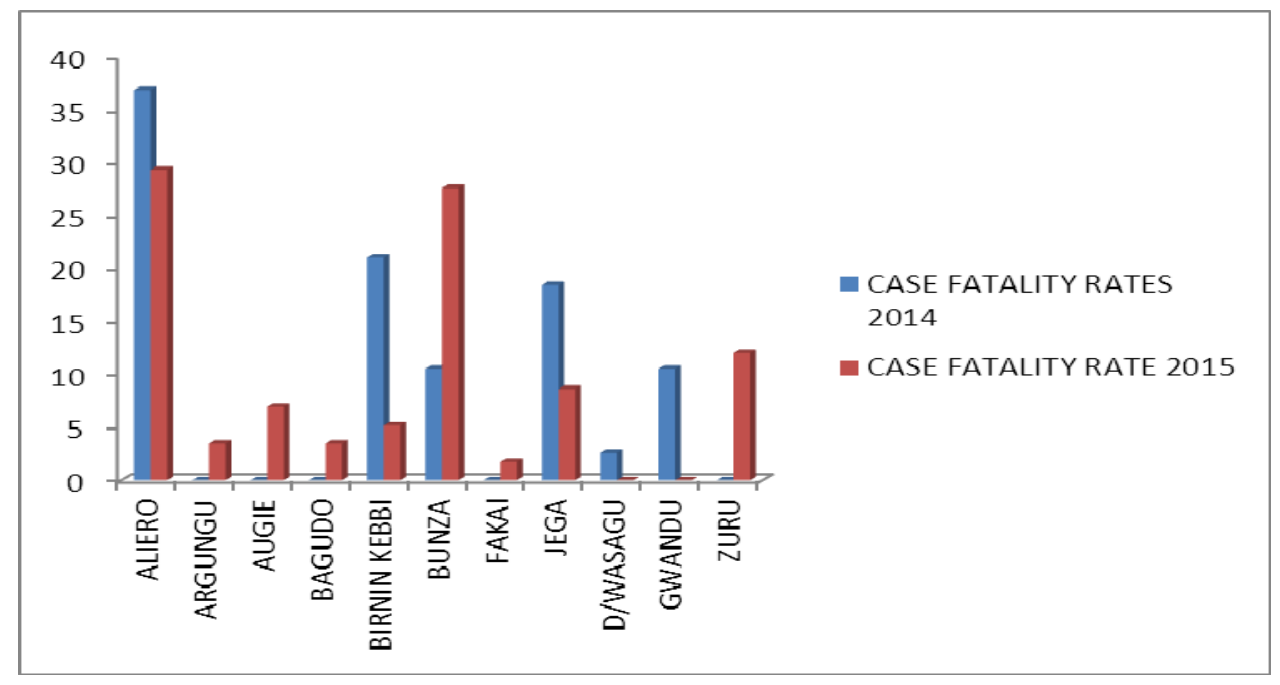

Figure 2. CSM Case fatality rates by LGA, Kebbi State (2014-2015)

Figure 3. Trend of CSM outbreak in Kebbi since 2011 from routine surveillance data

\begin{tabular}{llll}
\hline Year & Number of CSM cases reported through the year & Number of LGAs affected & CFR \\
\hline 2011 & 2 & 1 & 0 \\
2012 & 32 & 10 & 22 \\
2013 & 96 & 6 & 15 \\
2014 & 544 & 12 & 14 \\
2015 & 1992 & 16 & 5.5 \\
\hline
\end{tabular}

\section{Discussion}

Preventing and Controlling CSM Epidemic based on Findings from Laboratory Results and Field Experience.

\subsection{Strengthening Laboratory Capacity}

Limited number of samples is being processed due to challenges associated with sample collection (see Figure 3). Furthermore, none of the samples underwent culture studies in 2015 as against 2014, how much more polymerase chain reaction test. Similar challenges were pointed out in a recent publication authored by Bassey et al. (2016) where small numbers of CSF samples were taken and of course, even smaller numbers were processed in state laboratories in Northern Nigeria.

Obviously, Nigeria's quest to adequately manage CSM epidemic seems to have been limited by inadequate laboratory infrastructure. There is no designated public health laboratory for adequate investigation of CSM samples in Northern Nigeria. Most public health laboratories in the northwestern states are poorly funded, ill-equipped with limited human capacity to conduct molecular diagnosis (PCR). Therefore, the capacity to detect and fully characterize many epidemic prone-diseases including CSM is non-existent or at best described as weak.

Governments at both state and federal levels have not paid adequate attention to this seasonal epidemic. Commitment at the state level has been inconsistent and asynchronous with the federal government. However, WHO provides basic laboratory support for meningitis investigation-diagnostic kits such as Pastorex. CSF sample collection kits were also being supplied by the WHO to facilitate clinical sampling in some northern 
states during the epidemic. These are, however, often delivered late and inadequate (in number) or sometimes poorly managed by government official/healthworkers.

Furthermore, culture studies are not routinely done to understand potentially varied microbial profile responsible for the seasonal outbreak. This becomes germane for these reasons; there could be outbreak of mixed pathogenic cause and secondly, the antibiotics sensitivity profile of the pathogen can also be known via culture studies. As can be seen in 2014 data, $4.4 \%$ of the tested samples (cases) were either due to Streptococcus pneumoniae or Haemophilus influenza type $b$, underscoring the potential of outbreak of mixed pathogenic cause. This has implications on case management.

Consequently, a more coordinated approach by state and federal government and the partners is warranted, though WHO is currently attempting to foster a more coordinated and synchronous approach, in collaboration with Federal Ministry of Health and other relevant partner (Medecin San Frontieres, MSF) in the areas of laboratory analysis and related logistics ahead of future epidemics. Conceptually, laboratories are being identified where full laboratory analysis can be conducted on CSF samples. These laboratories are to be equipped by the government, though some support will be provided by health development partners. The challenge of this innovation is, however, that of logistics for transporting samples from the treatment camps and health facilities to a pooling centre (laboratory) in the state, and in turn to the identified central or zonal laboratory. Hence, state governments at this level should be committed to funding logistics involved in the interim.

\subsection{Paucity of Trained Medics to Take CSF Samples}

From Figure 2, 14\% of the suspected and confirmed cases had their CSF samples taken for laboratory analysis and this declined to $4 \%$ in 2015 . This reduction in the number of cases could be partly explained by paucity of trained medical personnel in the northern region, including, Kebbi State.

It is no longer news that Nigeria is bedeviled with brain drain which was abinitio pronounced in the academia but has permeated the medical profession in past three decades (Abejide, 2014). Arguably, Nigeria is the greatest "exporter" of medical professionals in sub-Sahara Africa, though, there are no reliable data regarding the number of doctors currently based and practising in Nigeria and in diaspora. The fluidity and poor documentation have made it challenging to adequately track doctors'mobility; hence, the precise number of doctors on ground in the country cannot be ascertained. At present, Nigeria has not met the recommended doctors/population ratio of one doctor to 600lives/person. This low doctor/population ratio also differs regionally and across states in Nigeria. Here in Kebbi State, there are not sufficient doctors to man hospitals, in fact, at the primary health care level, none of the health facility under the control of Local Government Area Council has a doctor working at this level while a General Hospital serving an entire Local Government has an average of 2-3 doctors managing the secondary health care centres. There are 243 medical doctors working in the state either as freelance (private) or employed by federal and state government for a population of over 4million. This is grossly inadequate how much more in the face of disease outbreak when some of these hospital-based medics are drafted to support treatment at designated camps and facilities.

Since the conduct of mass immunization campaign in November 2013, Nigeria has adopted and attempted to implement case-based surveillance for meningitis. However, paucity of medical doctors to conduct lumbar puncture procedure in the face of overwhelming number of cases has been a big challenge. Consequently, only very small number of samples ( $4 \%$ of total cases reported in 2015 versus $14 \%$ in 2014) could be taken and investigated at the laboratory. The number of cases that have laboratory analysis dwindled owing partly due to inadequate number of trained personnel (doctors) to conduct lumbar puncture procedure (clinical sampling). It is of note that that nurses are not allowed to take conduct lumbar procedure in Nigeria which is contrary to what is being practised in some neighbouring West African countries like Niger, Chad and Burkina Faso. This difference may be due to severe shortage of medical doctors in other West African countries. Some experts have posited that in the context of northern Nigeria, senior nurses or well-trained community health workers should be allowed to perform such minimally invasive procedure (conduct lumbar puncture procedure) in outbreak settings. However, this may be an interim measure, rather, the government at both levels (state and federal) should support training and motivate medical professionals through improved remuneration and provision of modern medical diagnostics and treatment facilities to improve retention in public services within the country.

\subsection{Weak Leadership and Governance}

There seems to be weak political visibility (between 2014 and 2015) during the periods of the outbreaks at the state level but sparingly available at the LGA level, with the exception of Jega and Aliero LGAs, where the LGA chairmen provided funds to support the renovation of outbreak treatment camps and associated logistics. Health 
workers were also mobilized from the primary health care facilities in these two worst hit LGAs.

Partners (WHO and MSF) contributed significantly to commodities supply for outbreaks control. Furthermore, huge doses of antibiotics were supplied as well as tetravalent vaccines for reactive vaccination in 2015 through International Coordinating Group for Meningitis Vaccines, ICG (WHO 2015). The state government also provided relatively limited support which was not timely and systematic, bearing in mind the problem of bureaucracy and accountability in public service. Emergency preparedness and responses (EPR) to outbreaks and other emergencies were essentially driven by development partners and no dedicated budget line known to the partners was available for funding response activities. It must be stated that leadership and coordination improved in 2015 comparatively to 2014 experience; however, it is still sub-optimal. The EPR serves as a coordinating team that provides leadership and governance for health emergencies. It has a technical component known as the Rapid Response Team while the larger EPR gives political and administrative support or direction. Sadly, the activities of these committees have been limited by poor political oversight, monitoring and accountability and, to a limited, extent funding.

Recently, health development partners, led by WHO have been able to support the state to revive and strengthen the EPR committee while funded workplan is being finalized in Kebbi State. Same is expected at the LGA but the present political and governance structure, specifically local government funding arrangement with state, i.e., inter-governmental relations, may impede optimal functioning at this level.

\subsection{Reduced Case Fatality Rate}

Case fatality reduced from $14 \%$ in 2014 to $4 \%$ in 2015 despite more cases reported. Improved social mobilization and community involvement might have impacted on health-seeking behaviours-improved the time of presentation at the treatment centres and hospitals. There seems to be improvement in accessibility to care (treatment camps set up in communities), availability of critical medical supplies, such as Ceftriaxone, which was made available by ICG through WHO, and also MSF to augment government supply. The 2015 response was perhaps better coordinated; there existed better working relationship between WHO, MSF and Government. This impacted positively on the effectiveness of the response to the outbreak in 2015, though, political campaign and electioneering process distracted the attention of political office holder at that time.

\subsection{Changes in Serotype-A Reflection of the Benefit of Mass Immunization and Call for Mass Immunization Campaign}

As a common practice in the African meningitis belt, reactive vaccinations are often conducted in response to seasonal meningitis outbreaks, and are usually conducted late (Greenwood, 1999; WHO, 2010), though this practice has recently changed as WHO now recommends that vaccination campaign be implemented as soon as possible, and within 4weeks of crossing the epidemic threshold (WHO, 2014). Hitherto, this (delayed) reactive vaccination has not impacted significantly in the prevention and control of meningitis in the region (Artal, 2015). However, in November 2013, there was a turn of event in Nigeria in the fight against type A Neisseria meningitidis which has been responsible for most of the outbreaks. A mass immunization campaign was conducted before the commencement of meningitis season, where type a conjugate vaccine was administered via fixed and mobile fixed post strategies, and the target age group was 1-29 years-representing about $70 \%$ of the population. The campaign was well accepted as it was long-awaited by many communities that have been repeatedly ravaged by seasonal meningitis outbreaks. It was indeed a successful campaign as shown by absence of type A serotype since 2013 (two consecutive years). As shown in Figure 2, 83\% of the samples tested were positive for Nm C in 2015 and 55\% were positive in 2014. No single Nm A has so far been reported in Kebbi State (even as at the time of writing the paper). Similar findings were observed in neighbouring northwestern states of Zamfara and Sokoto (Nigeria) and neighbouring West African countries such as Burkina Faso and Niger (Artal, 2015) that implemented mass vaccination campaign using conjugate vaccine against serotype A. These reported findings clearly show that mass immunization campaign, though expensive, appears to be cost-effective in the final analysis.

\section{Limitations of the Study}

The study made use of data generated from routine surveillance sourced from peripheral health facilities, treatment camps and big hospitals, however, some unknown proportion of the affected population might not have presented for orthodox treatment or may be missed by the conventional surveillance network. Therefore, the burden of disease may be higher than as reported by the government.

Smaller numbers of CSF samples were tested relative to the total number of cases reported in 2015 and also the inability to subject the CSF samples to further test such as culture and sensitivity or even PCR. This may be a 
drawback as there may be other pathogen such as Streptococcus pneumoniae or Haemophilus Influenza type b as seen in 2014. It could have been more informative, if such tests were conducted.

Another limitation of this study is a possibility of some unknown proportion of the population who might present similar symptomatology with CSM that have been classified as suspected cases since laboratory confirmation was not done for majority of the suspected cases. However, the aim of routine surveillance is to ensure that potential cases are not missed, hence the use of standard case definition for case identification which was recommended by WHO.

\section{Conclusion}

The seasonal epidemic of meningitis in Northern Nigeria-Kebbi, as an example, can be stemmed, if comprehensive epidemic prevention and control strategies are instituted. These strategies include improved surveillance and strengthening emergency preparedness and response, vigorous community mobilization, strengthening logistics and laboratory support, engagement and retention of more doctors in the public hospitals and more importantly, urgent implementation of well-planned mass immunization campaign (preventive), using a tetravalent vaccine (ACYW135). If all these are put together and effectively coordinated by government at all levels with support of health development partners like WHO and MSF, then the threat of type C epidemic will become negligible.

Lastly, effort to mitigate the impact of climate change must be looked at on the other hand as the transmissions of tropical diseases are aided by climate changes, which are essentially triggered by socio-anthropological activities in the quest for economism and technological advancement, even in the developing countries of Africa.

\subsection{Notes from Thee Paper}

1) Serotype $C$ is the new major pathogenic cause in Kebbi State and Northwestern Nigeria for epidemic for two consecutive years, post mass vaccination.

2) Impact of mass vaccination campaign has been palpable considering the fact that no serotype $A$ has been reported in Northern Nigeria for two consecutive years (since November 2013) after mass vaccination using conjugate vaccine against serotype A which caused majority of epidemic before this period

3) A call for holistic response to the epidemic- sustaining surveillance, need to improve laboratory support, strengthened governance through a strong and responsive EPR teams and implementation of mass vaccination campaign against type $\mathrm{C}$ (which is cost effective).

4) Mass preventive vaccination is strongly recommended, and concurrently, effort to mitigate the impact of climate change should be considered as the meningitis belt has been expanding in Nigeria in the past 3years

\section{Competing Interests Statement}

The authors declare that there is no conflict of interests regarding the publication of this paper.

\section{References}

Abejide, L. E. O. (2014). A Spatio-Temporal Analysis of Migration of Highly Skilled Professionals from Health Institutions in Nigeria. Journal of African Studies and Development, 6(5), 95-112. http://dx.doi.org/10.5897/ JASD2013.0267

Artal, F. J. C. (2015). Meningoccal meningitis: Vaccination outbreak response and epidemiological changes in the African meningitis belt. Int Health, 7, 226-227. http://dx.doi.org/10.1093/inthealth/ihv025

Bassey, E. B., Vaz, R. G., Gasasira, A. N., Braka, F., Weldegriebriel, G., Komakech, W., \& Okocha-Ejeko, A. (2016). Pattern of the meningococcal meningitis outbreak in Northern Nigeria. Int. J of Infect Dis, 43, 62-67. http://dx.doi.org/10.1016/j.ijid.2015.12.016

Federal Ministry of Health (Nigeria)/World Health Organisation. (2016). Stakeholders' Meeting on CSM LaboratorySurveillance@Sokoto State, Nigeria, 3-4th February, 2016.

Greenwood, B. (1999). Manson Lecture: Meningococcal Meningitis in Africa. Trans R Soc Trop Med Hyg, 93, 341-353. http://dx.doi.org/10.1016/S0035-9203(99)90106-2

Marc LaForce, F., Ravenscroft, N., Djingarey, M., \& Viviani, S. (2009). Epidemic Meningitis due to Group a Neisseria meningitides in the African meningitis belt: A persistent problem with imminent solution. Vaccines, 27S, B13-B19.

Molesworth, A. M., Cuevas, L. E., Connor, S. J., Morse, A. P., \& Thomson, M. C. (1993). Environmental Risk and Meningitis Epidemics in Africa. Emer Infec Dis, 10(9), 1287-1293. 
World Health Organisation. (2010). Meningitis in Chad, Niger and Nigeria: 2009 epidemic season. Wkly Epidemiol Rec, 8, 57-68.

World Health Organisation. (2014). Meningitis Outbreak Response in Su-Saharan Africa: WHO Guideline. World Health Organisation, Geneva.

World Health Organisation. (2015). Highly contagious meningitis outbreak continues in African countries. Retrieved February 26, 2016, from http://www.afro.who.int/en/media-centre/afro-feature/item/7579-highly -contagious-meningitis-outbreaks-continue-in-african-countries.html

World Health Organisation. (2016). Immunisation, Vaccines and Biologicals. Retrieved January 13, 2016, from http://www.who.int/immunization/monitoring_surveillance/burden/vpd/surveillance_type/sentinel/meningiti s_surveillance/en/

Yves, T., Berthe-Marie, N.-L., Kokou-Louis-Sewonou, A., Mathilde, L., Seydou, Y., Boubacar, N., \& Bradford, D. G. (2006). The rise and fall of epidemic Neisseria meningitides Serogroup W135 meningitis in Burkina Faso, 2002-2005. Clin Infect Diseases, 43, 817-822. http://dx.doi.org/10.1086/507339

\section{Copyrights}

Copyright for this article is retained by the author(s), with first publication rights granted to the journal.

This is an open-access article distributed under the terms and conditions of the Creative Commons Attribution license (http://creativecommons.org/licenses/by/3.0/). 